ARTICLE

\title{
Ring-opening functionalizations of unstrained cyclic amines enabled by difluorocarbene transfer
}

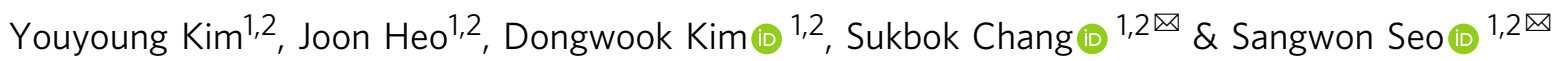

Chemical synthesis based on the skeletal variation has been prolifically utilized as an attractive approach for modification of molecular properties. Given the ubiquity of unstrained cyclic amines, the ability to directly alter such motifs would grant an efficient platform to access unique chemical space. Here, we report a highly efficient and practical strategy that enables the selective ring-opening functionalization of unstrained cyclic amines. The use of difluorocarbene leads to a wide variety of multifaceted acyclic architectures, which can be further diversified to a range of distinctive homologative cyclic scaffolds. The virtue of this deconstructive strategy is demonstrated by successful modification of several natural products and pharmaceutical analogues.

\footnotetext{
${ }^{1}$ Department of Chemistry, Korea Advanced Institute of Science and Technology (KAIST), Daejeon 34141, Republic of Korea. ${ }^{2}$ Center for Catalytic

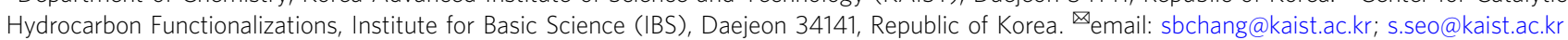


G iven the ubiquity of cyclic amines in a myriad of natural products and synthetic compounds, a growing emphasis has been placed on how we harness and manipulate such prestigious motifs for the construction of value-added molecules ${ }^{1-4}$. While significant progress has been made in the development of appending processes for their peripheral variation ${ }^{5-10}$, the skeletal diversification by means of ring-opening, -contraction, -expansion or -fusion approaches remains limited (Fig. 1a) ${ }^{11}$. This is particularly pertinent for the unstrained cyclic systems, which is attributed to the fact that the structural reorganization is inevitably accompanied by highly demanding $\mathrm{C}-\mathrm{N}^{12,13}$ or $\mathrm{C}-\mathrm{C}^{14-16}$ bond cleavage. To this end, the ability to access these robust $\sigma$-bonds in an efficient and selective manner would provide a powerful handle for the diversification of azacyclic skeletons.

Despite recent advances in the realm of $\mathrm{C}-\mathrm{C}$ bond transformations ${ }^{17-20}$, the selective cleavage of $\mathrm{C}-\mathrm{N}$ bonds would be a compelling strategy for ring-opening functionalization of cyclic amines. Nonetheless, the process that cleaves and turns $\mathrm{C}-\mathrm{N}$ bonds into versatile functionalities is still relatively scarce. For instance, the conventional reductive approach, typically employing molecular hydrogen in combination with transition metal catalysts, often leads to non-transformable hydrocarbon frameworks by reductive $\mathrm{C}-\mathrm{N}$ bond cleavage, thus limiting the degree of scaffold diversity (Fig. 1b, top $)^{21-24}$.

In an ideal scenario, the ring-opening reaction would be followed by the installation of versatile motifs that can be further transformed for diversity-generating chemical synthesis ${ }^{25-27}$. With this regard, the oxidative strategy has enjoyed methodological prosperity in recent years ${ }^{28-32}$. In a classical reaction mode, oxidation of the $\alpha$-carbons of cyclic amines generates labile hemiaminals, thereby enabling the conversion of otherwise inert $\mathrm{C}-\mathrm{N}$ bonds into amino-aldehyde or -carboxylic acid moieties (Fig. 1b, middle). While the conventional oxidative strategies often suffer from low functional group compatibility, improved procedures have been recently developed by harnessing transition metals. A conspicuous example includes the elegant work by Sarpong ${ }^{33}$, which furnishes a deconstructive halogenation of cyclic amines via cascade decarboxylative process.

An alternative way of rendering the $\mathrm{C}-\mathrm{N}$ bonds more accessible is through the formation of quaternary ammonium salts (Fig. 1b,

a

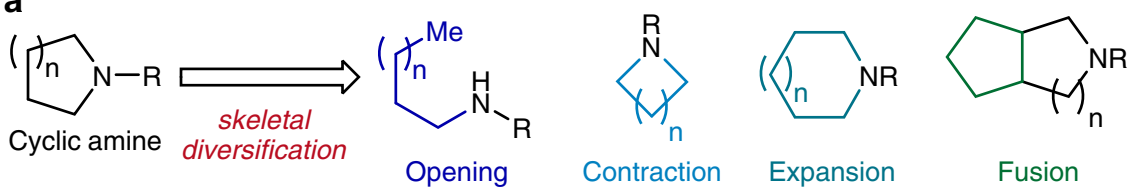

- Manipulate the biological properties with variation of the overall molecular shape
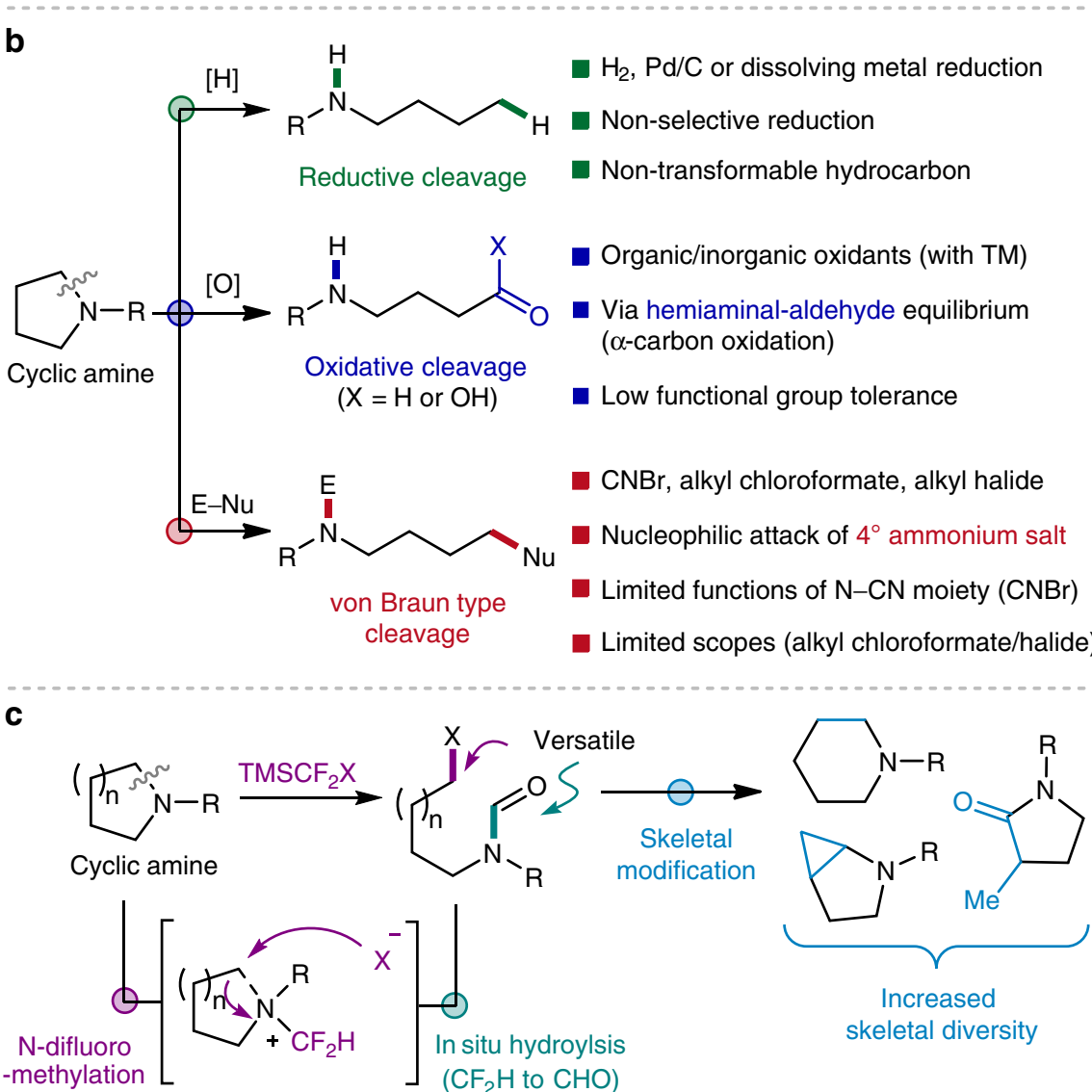

-methylation [ $\left(\mathrm{CF}_{2} \mathrm{H}\right.$ to $\left.\mathrm{CHO}\right)$

Mild \& selective N-difluoromethylation

Broad scope over various cyclic amines

Diversification based on the reactivities at both halide and $\mathrm{N}$-formyl groups

Fig. 1 C-N Bond cleavage of unstrained cyclic amines. a Importance of skeletal diversification of unstrained cyclic amines. b Previously reported strategies for C-N bond cleavage of cyclic amines. c This work: Ring-opening halogenation via N-difluoromethylative C-N bond cleavage. 
bottom). The increased bond polarity ameliorates the leaving group ability of amino moiety, ultimately allowing the $\mathrm{C}-\mathrm{N}$ bond cleavage by means of elimination ${ }^{34}$ or nucleophilic substitution reactions ${ }^{35-37}$. This chemistry has been widely utilized in the ringopening reaction of strained rings ${ }^{38-42}$, however, its use in unstrained cyclic systems is somewhat thwarted by the stability of the involved salts. Of a great potential for further development is the von Braun reaction, which employs cyanogen bromide $(\mathrm{CNBr})$ in ring-opening bromination of azacyclic compounds ${ }^{43,44}$. This reaction outperforms other strategies in terms of efficiency and applicability, but has found relatively narrower synthetic applications ${ }^{45-48}$. This is mainly due to the limited function of the resulting $\mathrm{N}-\mathrm{CN}$ group and the requirement of harsh conditions for its removal. Additionally, cyanogen bromide is highly toxic, and can undergo explosive trimerization to cyanuric bromide or decompose to hazardous hydrogen cyanide $(\mathrm{HCN})^{49}$. Chloroformates have been utilized as milder reagents ${ }^{50-54}$, however generally applied for strained ring systems ${ }^{38,41}$, with an exceptional report by Cho still limited to $\mathrm{N}$-alkyl-substituted 5 -membered cyclic amines ${ }^{55}$. One of the main challenges associated with such reagents comes from a poor capability to access the key ammonium salt intermediates, which in turn restricts the employable ring types.

Here we report a highly efficient and practical strategy for the ring-opening functionalization of a wide variety of unstrained cyclic amines, including but not limited to 5- and 6-membered rings (Fig. 1c). The use of difluorocarbene allows a prominently selective $\mathrm{N}$-difluoromethylation, with subsequent ring-opening halogenation and in situ hydrolysis furnishing acyclic $\mathrm{N}$-formyl haloamines. The resulting pluripotent motifs further enable the construction of diverse arrays of homologated scaffolds by transforming both $\mathrm{N}$-formyl and halide functional groups incorporated in products, providing a unique platform for scaffold diversity that cannot be accessed by the conventional von Braun-type reactions. Not only a wider range of cyclic amines can be deconstructively modified, but this strategy can also be applied to complex molecules bearing labile functional groups.

\section{Results}

Reaction development. Difluorocarbene serves as an excellent reagent in transition metal-free cyclopropanation of alkenes and alkynes, and can also react with various carbon or heteroatom nucleophiles ${ }^{56,57}$. We envisaged that its highly-electrophilic character would permit an efficient and selective generation of key ammonium salt intermediates from a variety of azacyclic cores, whereby the introduced difluoromethyl $\left(\mathrm{CF}_{2} \mathrm{H}\right)$ group would then manipulate the ring properties to eventually enable the deconstructive functionalization of challenging $\mathrm{C}-\mathrm{N}$ bonds. Difluorocarbene can be readily generated by $\alpha$-elimination of $\mathrm{CF}_{2} \mathrm{XY}$-type reagents, among which (bromodifluoromethyl)trimethylsilane $\left(\mathrm{TMSCF}_{2} \mathrm{Br}\right.$ ) stands out as one of the most practical precursors ${ }^{58}$. It is non-toxic, air- and moisture-stable, easy to handle, and can generate the reactive species under mild conditions.

We, therefore, focused on the use of $\mathrm{TMSCF}_{2} \mathrm{Br}$ in our initial investigation towards the deconstructive functionalization of $\mathrm{N}$ phenylpyrrolidine 1a. Pleasingly, after extensive screenings of various reaction parameters, we identified the optimized conditions consisting of $\mathrm{TMSCF}_{2} \mathrm{Br}, \mathrm{NH}_{4} \mathrm{OAc}$ base and 1,2-dichloroethane (1,2-DCE) solvent, which furnished the ring-opening product $2 \mathbf{a}$ in $85 \%$ yield at room temperature in $12 \mathrm{~h}$ (Fig. 2a, entry 1 ). Interestingly, the deconstructive product was incorporated with an $\mathrm{N}$-formyl moiety, presumably by in situ hydrolysis of the unstable $\mathrm{N}-\mathrm{CF}_{2} \mathrm{H}$ group ${ }^{59}$. This cascade process is beneficial in synthetic aspects, as the $\mathrm{N}$-formyl group not only is readily removable ${ }^{60}$, but also provides a unique opportunity for diverse post-modifications. Control experiments established the importance of base (entry 2), which is required for the generation of difluorocarbene intermediate. Notably, an alternative difluorocarbene source (entry 3 ), a dichlorocarbene variant ${ }^{61}$ (entry 4 ) and the conditions developed by $\mathrm{Cho}^{55}$ (entry 5) were found to be incompetent (see Supplementary Table 1 for details). Encouraged by the above promising result, we also examined the feasibility of $\mathrm{N}$-ethylpyrrolidine $\mathbf{1 b}$ in the ring-opening bromoformylation (Fig. 2b). We could obtain the presupposed intermediacy of quaternary ammonium salt by treating $\mathbf{1 b}$ under the optimized conditions at room temperature (int1, 78\%). A moderate heating of the isolated intermediate int1 consequently resulted in a facile ringcleavage to afford $\mathbf{2 b}(83 \%)$.

Substrate scope of 5-membered rings. Having established the optimal conditions, we next evaluated the substrate scope over a range of 5-membered azacyclic compounds (Fig. 3). All substrates examined were smoothly reacted under mild conditions irrespective of the nature of $\mathrm{N}$-substituents, furnishing the ringopening bromoformylation products in excellent selectivity and high efficiency (2a-2r). Noteworthy is that 1.1 equiv. of the difluorocarbene precursor was sufficient enough for $\mathrm{N}$-alkyl substrates (2b-2c). Methoxy, trifluoromethyl, ketone and amide functionalities on $N$-aryl groups were found to be well-tolerated in ring-opening of pyrrolidines (2d-2g, 77-91\%). Benzo-fused and fully saturated bicyclic amines were also effectively transformed $(\mathbf{2 h}-\mathbf{2 l}, \mathbf{5 0}-83 \%)$. In case of octahydroindolizine (1k), the skeleton was ring-opened exclusively at the 5-membered unit. A dihydropyrrole $\mathbf{1 m}$ was readily ring-opened without affecting the olefin moiety $(\mathbf{2 m}, 86 \%)$, proving its orthogonality to the previously reported cyclopropanation reactions ${ }^{58}$.

Significantly, reactivity differentiation by basicity of amines or steric influences allows the reaction to occur in excellent regioselectivities. For instance, when there are two nitrogen atoms present in the same or different azacyclic skeletons, those with a more basic amine were exclusively difluoromethylated and subsequently ring-opened ( $2 \mathbf{n}$ and $\mathbf{2 o}, 81$ and $72 \%$ ). For $2-$ or $3-$ substituted pyrrolidines, the ring was selectively cleaved at the sterically less demanding 5-position (2p-2r, 60-95\%). We were delighted to find that the variation of halides was also possible, affording the chlorination or iodination products in moderate to good yields (2a-Cl, 2b-Cl and 2d-I, 51-82\%). In addition, the ring-opening acetoxylation was achieved (2a-OAc, 70\%) by employing an excessive amount of $\mathrm{NH}_{4} \mathrm{OAc}$ in combination with (chlorodifluoromethyl)trimethylsilane $\left(\mathrm{TMSCF}_{2} \mathrm{Cl}\right)$.

Elucidation of the regioselectivity in $\mathrm{C}-\mathrm{N}$ bond cleavage. The finding of a convenient method for ring-opening functionalization of 5-membered cyclic amines led us to probe the origin of selectivity for $\mathrm{N}$-alkyl substrates (Fig. 4). Taking a $\mathrm{CF}_{2} \mathrm{H}$ salt int1 as a model intermediate, three potential substitution pathways were computationally evaluated (Fig. 4a). Transition state analysis was consistent with the observed selectivity for int 1 . The ring-opening process (TS3) displayed an activation barrier of $20.7 \mathrm{kcal} / \mathrm{mol}$, whereas those for deethylation (TS2) and dedifluoromethylation (TS1) were higher in energy by 5 and $17 \mathrm{kcal} / \mathrm{mol}$, respectively. Although the product from the ringopening pathway (RO1) was slightly uphill by $2.6 \mathrm{kcal} / \mathrm{mol}$, this process was expected to be facilitated by the subsequent hydrolysis of the $\mathrm{N}-\mathrm{CF}_{2} \mathrm{H}$ group to the thermodynamically stable $N$-formyl product $\mathbf{2 b}$ (see Supplementary Fig. 3). The revealed preference of intl to undergo the ring-opening pathway (TS3) over $N$-deethylation (TS2) could be quantified by a distortioninteraction analysis (Fig. 4b). Along with the intrinsic reaction coordinate, distortion energy term of ring-opening is significantly 
a

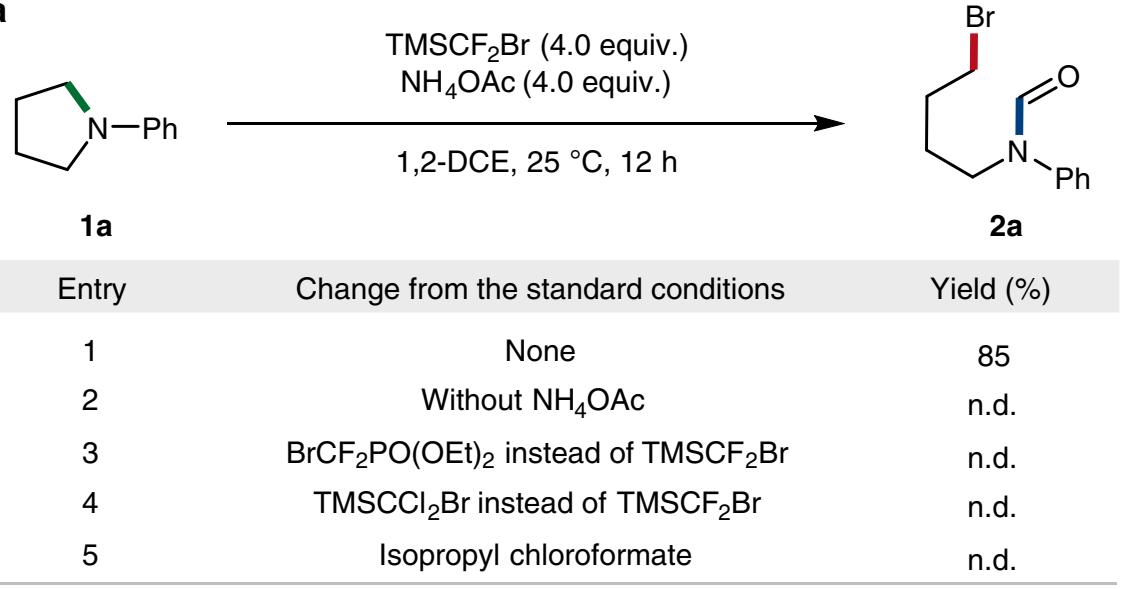

b

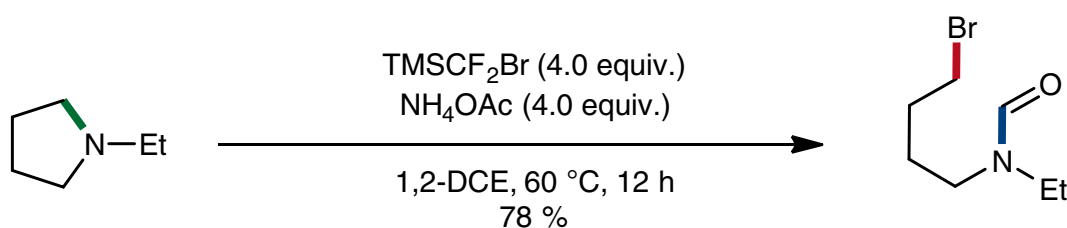

16

$$
78 \%
$$

$2 b, 83 \%$
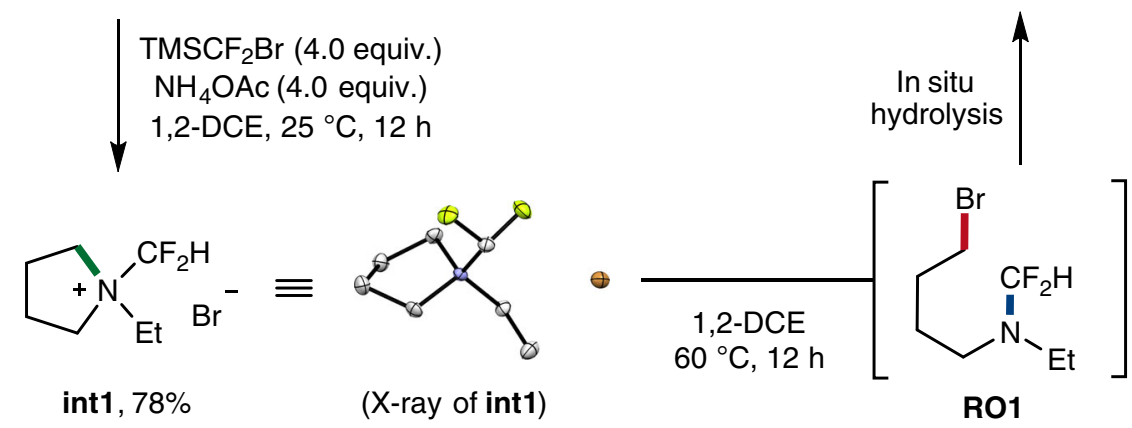

Fig. 2 Reaction development. a Selected optimization conditions for the ring-opening bromoformylation of $\mathrm{N}$-phenylpyrrolidine 1a; All reactions were performed on a $0.2 \mathrm{mmol}$ scale; 1,2-DCE, 1,2-dichloroethane; n.d., not detected; Isolated yields. $\mathbf{b}$ Isolation of an ammonium salt intermediate from $\mathrm{N}$ ethylpyrrolidine $\mathbf{1 b}$

smaller than that of $\mathrm{N}$-deethylation, whereas their interaction energy profiles are rather similar. From these outcomes, it could be concluded that the earlier nature of the ring-opening transition state with respect to the $\mathrm{C}-\mathrm{N}$ breaking event is responsible for the corresponding selectivity. Moreover, the DFT-optimized structures of int1 clearly show that the cyclic $\mathrm{C}-\mathrm{N}$ bond is noticeably longer than the ethyl $\mathrm{C}-\mathrm{N}$ bond presumably because of the ring strain, indicating that the attack of the cyclic $\mathrm{C}-\mathrm{N}$ bond involves the least structural reorganization towards the corresponding transition state (Fig. 4c).

Extension to 6-membered cyclic amines. With the comprehensive results obtained for the ring-opening functionalization of five-membered cyclic amines, we wondered whether the current methodology could also be applied to 6-membered cyclic amines. Delightfully, the initial attempt with $N$-phenylpiperidine $\mathbf{3 a}$ as a substrate gave a ring cleavage product $4 \mathbf{a}$ under the optimized conditions at $60{ }^{\circ} \mathrm{C}(80 \%$, Fig. 5a). On the other hand, $\mathrm{N}$-alkyl substrates were obstructed by undesired $\mathrm{N}$-dealkylation, which has been a major problem encountered in most of the existing ring-opening strategies ${ }^{31}$. For instance, $N$-ethylpiperidine $\mathbf{3 b}$ afforded the ring-opening product $\mathbf{4 b}$ in $18 \%$ yield along with the $N$-dealkylation product 5 in $82 \%$ yield (4b:5, 1:4.6). In addition to the earlier example of octahydroindolizine (1k, Fig. 3), these results suggested again that 6-membered rings are less influenced by the presence of a $\mathrm{CF}_{2} \mathrm{H}$ group, presumably due to their inherent ring stability. Intriguingly, we discovered that this issue could be overcome by examining the steric influence of the $\mathrm{N}$ alkyl group. In particular, the ring-opening pathway becomes substantially more favourable with increment of substituents at the $\gamma$-position (4d-4f). For example, $N$-dealkylation pathway was largely suppressed in the case of N-neohexyl substrate (3f), which exclusively gave the ring-opening product 4 ff in $86 \%$ yield. Meanwhile, $N$-isobutyl substrate (3c) showed a similar selectivity to $N$-ethyl substrate $(\mathbf{3 b})$, indicating that a $\beta$-substituent has a negligible effect on the selectivity. These outcomes are captivating as the observed trend is opposed to that of general $\mathrm{S}_{\mathrm{N}} 2$ reactions, in which branching carbons farther away from the electrophilic site tend to have a smaller effect on the nucleophilic attack ${ }^{62}$.

Having identified this interesting steric influence, we further sought to investigate the observed selectivity by computational analysis. $N$-Difluoromethyl- $N$-ethyl piperidinium bromide (int2) and the analogous $N$-neohexyl salt (int3) were representatively chosen for the case study. Consistent with the experimental results, the DFT calculations on the $\mathrm{N}$-ethyl salt int2 show that $\mathrm{N}$ dealkylation is marginally preferred over the ring-opening pathway $\left(\Delta \Delta G^{\ddagger}=1.6 \mathrm{kcal} / \mathrm{mol}\right.$, Fig. $\left.5 \mathrm{~b}\right)$. In sharp contrast, the activation barrier for ring-opening of int 3 is $4.6 \mathrm{kcal} / \mathrm{mol}$ lower in energy than 


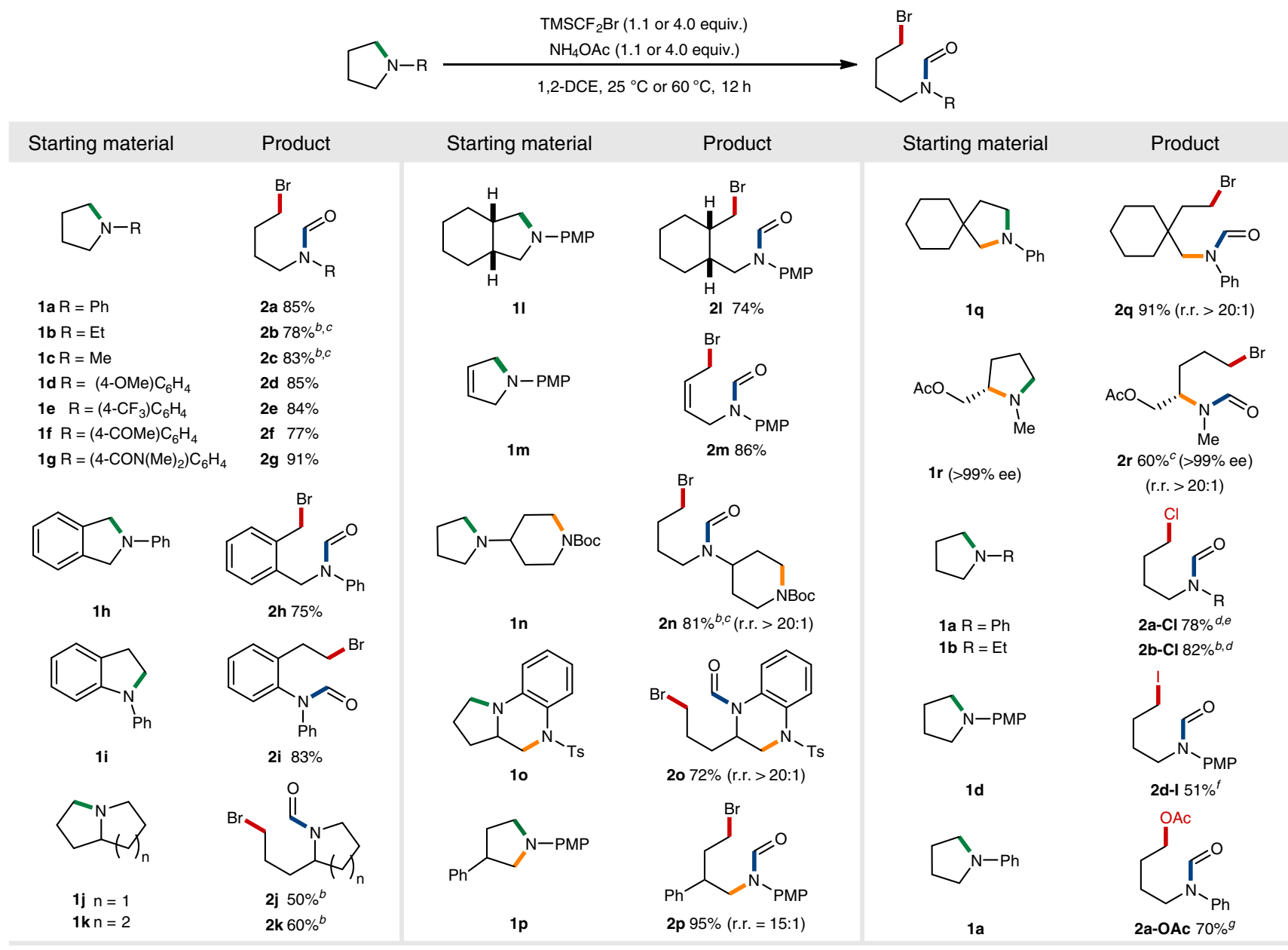

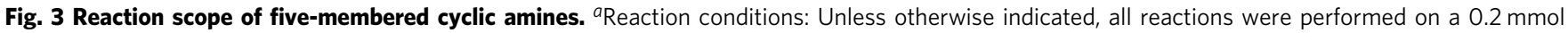
scale using cyclic amine (1.0 equiv.), $\mathrm{TMSCF}_{2} \mathrm{Br}$ (4.0 equiv.), $\mathrm{NH}_{4} \mathrm{OAc}$ (4.0 equiv.) and 1,2-DCE ( $\left.0.5 \mathrm{~mL}\right)$ at room temperature. regioselectivity ratios (r.r.) were determined by ${ }^{1} \mathrm{H}$ NMR spectroscopy of crude mixtures. ${ }^{b} 60^{\circ} \mathrm{C} .{ }^{c} 1.1$ equiv. of $\mathrm{TMSCF}_{2} \mathrm{Br}$ and 1.1 equiv. of $\mathrm{NH}_{4} \mathrm{OAc}$. ${ }^{d} \mathrm{TMSCF}_{2} \mathrm{Cl}(4.0$ equiv.) instead of $\mathrm{TMSCF}_{2} \mathrm{Br}$. ${ }^{\mathrm{e}} \mathrm{KF}$ (4.0 equiv.) as an alternative base, with $\mathrm{H}_{2} \mathrm{O}\left(0.05 \mathrm{~mL}\right.$ ) as a co-solvent. $\mathrm{TTMSCF}_{2} \mathrm{I}$ (4.0 equiv.) instead of $\mathrm{TMSCF}_{2} \mathrm{Br}$. 94.0 equiv. of $\mathrm{TMSCF}_{2} \mathrm{Cl}$ and 16.0 equiv. of $\mathrm{NH}_{4} \mathrm{OAc}$. PMP para-methoxyphenyl.

the corresponding $N$-dealkylation (Fig. $5 \mathrm{c}$ ). The structural analysis of the intermediates and their transition states further revealed the primary steric influence by $\gamma$-substituents (Fig. 5d). The DFToptimized structure of the $N$-neohexyl salt int3 clearly shows that the substituent at the $\gamma$-position of $N$-alkyl group considerably disturbs the $\mathrm{N}$-dealkylation path. As a result, the rotation along the $\mathrm{C}(\alpha)-\mathrm{C}(\beta)$ bond becomes imperative as the nucleophile approaches in the transition state, which then causes an unfavourable steric repulsion with the $\mathrm{N}-\mathrm{CF}_{2} \mathrm{H}$ piperidine moiety. Consequently, $\mathrm{N}$-dealkylation becomes disfavoured with a significantly increased activation barrier, rendering the ring-opening pathway relatively more favourable.

Substrate scope of 6-membered and larger rings. With the obtained understanding of reactivity and selectivity, a range of 6membered cyclic amines were explored for the current ringopening functionalization strategy (Fig. 6a). Piperidine rings were viable to give the desired products in good to excellent yields (4a-4i, 79-99\%), and both $N$-aryl and $N$-alkyl substituents could be incorporated. Ester functional groups were found to be tolerable, with the 4 -substituted piperidines ( $\mathbf{3 g}$ and $\mathbf{3 h}$ ) giving rise to $\gamma$ - and $\delta$-amino esters $\mathbf{4 g}$ and $\mathbf{4 h}$ in 85 and $80 \%$ yields, respectively. A spiro bicyclic substrate (3i) was also effectively functionalized to give the corresponding product $4 \mathbf{i}$ in $99 \%$ yield.
Morpholine was also feasible with both $N$-phenyl (3j) and $N$ neohexyl (3k) substituents, affording the valuable acyclic products $\mathbf{4 j}$ and $\mathbf{4 k}$ in excellent yields (90\%). 1-Phenyl-4-tosylpiperazine (31) was selectively ring-opened at the 2-position (4l, 76\%, r.r. $>20: 1)$, which was enabled by a selective difluoromethylation at the nitrogen incorporated with a phenyl group. Tetrahydroisoquinoline substrate (3m) was reacted at the more electrophilic benzylic site to give the ring-opened product in an excellent yield and high selectivity $(4 \mathrm{~m}$, $93 \%$, r.r. = 12:1). The reaction of trans-decahydroisoquinoline (3n) was also highly selective, leading to the deconstructive product by bromination at the sterically less demanding site (4n, $88 \%$, r.r. $>$ 20:1). Moreover, julolidine (3o) and quinuclidine (3p) cyclic cores were effectively transformed to the corresponding bromoamines (4o and 4 p, 71 and $75 \%$, respectively).

Notably, arecoline $\mathbf{3 q}$ exclusively gave the ring-opening product 4q $(87 \%)$ by a Hofmann-type elimination reaction. This relatively simple natural product had previously been reported to favour $N$-demethylation when treated with chloroformates ${ }^{51}$. The reaction of an $\mathrm{N}$-butylpiperidine substrate $3 \mathbf{r}$ occurred exclusively at the position appended with a phenyl group (4r, 87\%). These two examples showed that steric at the $N$-alkyl group is not necessary in cases where the ring is substituted with certain functional groups. Furthermore, the reaction was also found feasible with 7 - and 8-membered rings, furnishing the desired linear bromoamine 
a

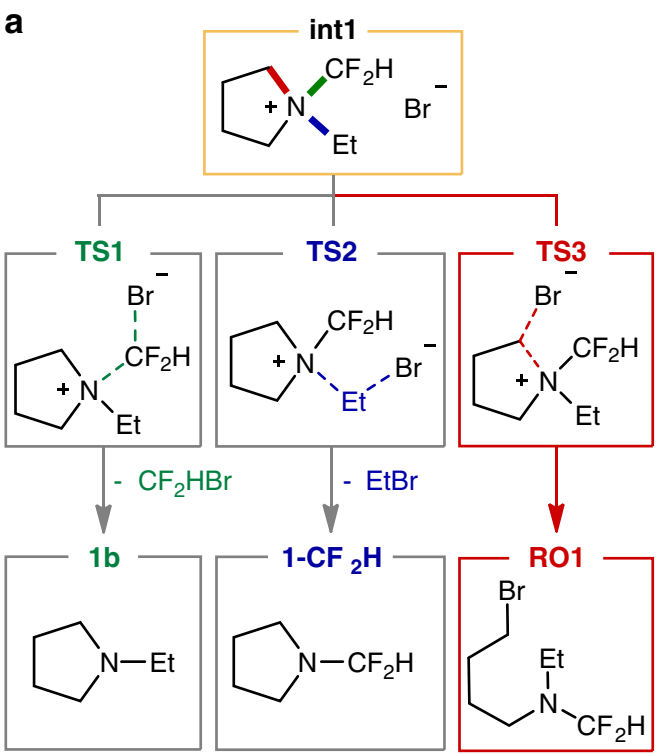

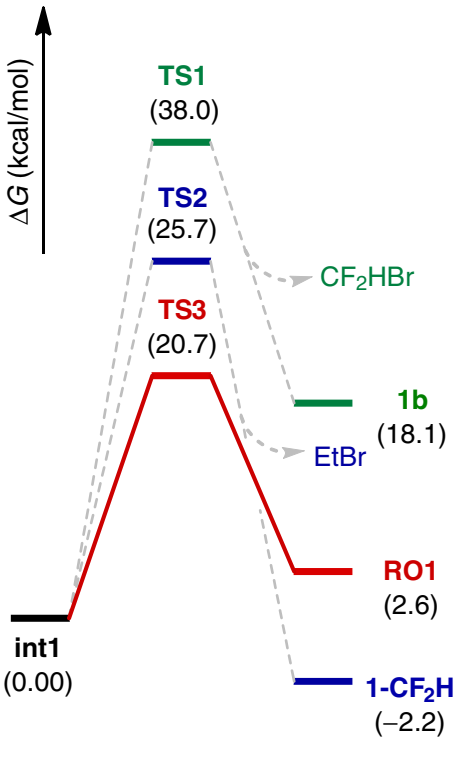

C

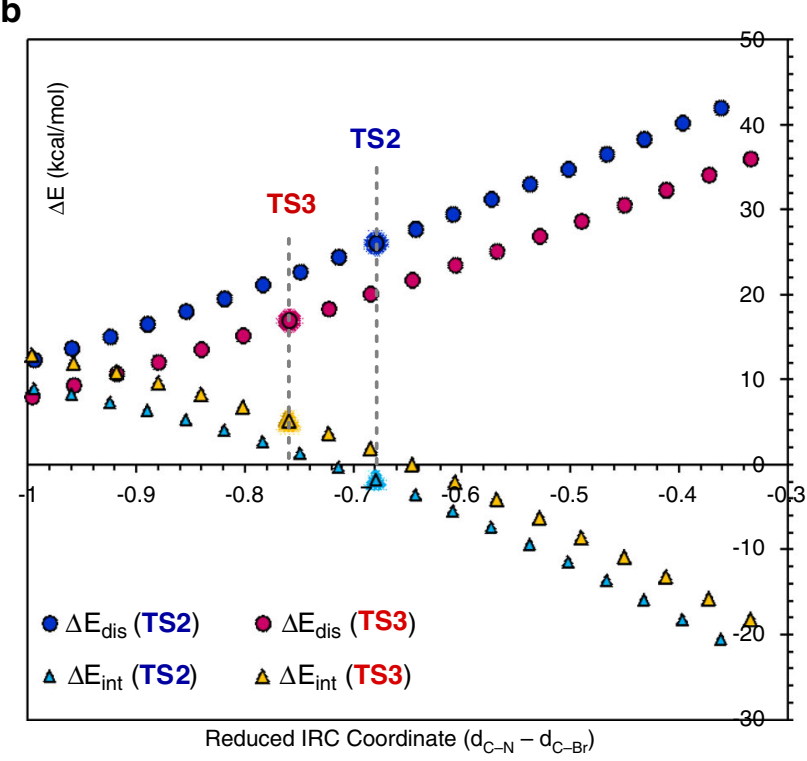

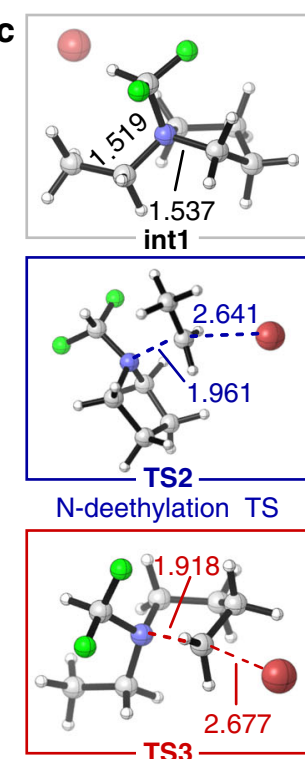

ring-opening TS

Fig. 4 Evaluation of selectivity for 5-membered cyclic amines with DFT calculation. a Reaction energy profiles of three possible nucleophilic substitution pathways from $\mathrm{N}-\mathrm{CF}_{2} \mathrm{H}$ salt (int1); green: dedifluoromethylation path leading to $\mathbf{1 b}$ (via TS1), blue: deethylation path leading to $\mathbf{1}-\mathbf{C F}_{\mathbf{2}} \mathbf{H}$ (via $\mathrm{TS}_{2}$ ), and red: ring-opening path leading to RO1 (via TS3). b Distortion-interaction analysis along IRC coordinate of int1; blue dot: distortion energy of deethylation, red dot: distortion energy of ring-opening, sky blue triangle: interaction energy of deethylation, and yellow triangle: interaction energy of ring-opening. c DFToptimized structures of int1 and its transition states for deethylation (blue, TS2) and ring-opening (red, TS3).

products in excellent yields (7a-7b and $\mathbf{9 a - 9 b}, 68-95 \%)$. Overall, the ability to manipulate various cyclic skeletons allowed the synthesis of difficult-to-access acyclic bromoamine motifs with a wide range of $\mathrm{N}$-substituents, including those previously underexplored for 6-membered or larger cyclic systems.

To further highlight the broad applicability of our method, late-stage modifications of biologically relevant compounds were examined (Fig. 6b). The exceptional functional group tolerance and excellent regioselectivity were proven with a Sildenafil analogue 10 that contains various nitrogen atoms, which afforded 11 by an exclusive ring-opening of the piperazine scaffold in $43 \%$ yield along mostly with unreacted starting material. Reserpine also underwent an efficient $\mathrm{C}-\mathrm{N}$ bond cleavage by acetoxylation at the benzylic site, leading to the synthetically challenging 10-membered azacyclic product 12 (56\%, >20:1 d.r.).
Notably, Fenpiprane and Prozapine, analogous drugs correspondingly containing $N$-alkyl substituted 6- and 7-membered cyclic amines, selectively afforded the ring-opening products $\mathbf{1 3}$ and 14 in 60 and $81 \%$ yields, respectively. Likewise, $(S)-(+)$-citronellyl morpholine was effectively ring-opened to give $\mathbf{1 5}$ in $63 \%$ yield. Considering that the basic motifs of these examined compounds are vastly present in many other pharmaceuticals and natural products, this approach is expected to find wide synthetic applications.

Skeletal diversification. In addition to the excellent substrate generality and applicability demonstrated above, we envisioned that our resulting product could be further used as a pluripotent intermediate for skeletal diversification. The substrate-based strategy relying on the pre-installation of an internal functional group would limit the obtainable scaffolds from a specific ring system. With this 
a

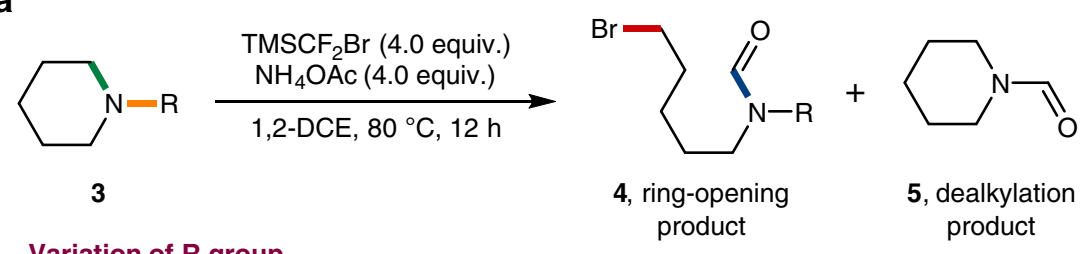

Variation of $\mathbf{R}$ group

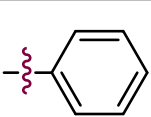

3a

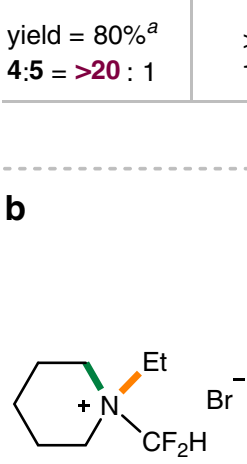

int2 (0.0)

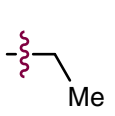

3b<smiles>CCC(C)C</smiles>
$3 c$

$>95 \%$

$1: 5.5$

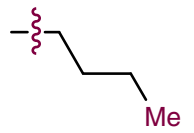

3d<smiles>CC(C)CCC(C)C</smiles>

$3 e$

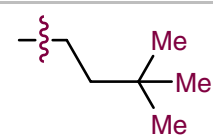

$3 f$

$1: 1.7$

$>95 \%$

$1.8: 1$

$86 \%$

$>20: 1$

Yields determined by ${ }^{1} \mathrm{H}$ NMR analysis of crude mixtures; ${ }^{a}$ Run at $60^{\circ} \mathrm{C}$

b

Ring-opening TS

$(25.4 \mathrm{kcal} / \mathrm{mol})$

(From int2, 4:5 = 1:4.6)

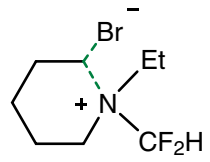

Not favoured,

but allowed
$\Delta \Delta G^{\ddagger}=$

$1.6 \mathrm{kcal} / \mathrm{mol}$
$\mathrm{N}$-deethylation TS

$(23.8 \mathrm{kcal} / \mathrm{mol})$

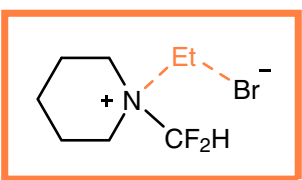

favoured

(From int3, 4:5 = >20:1)

C

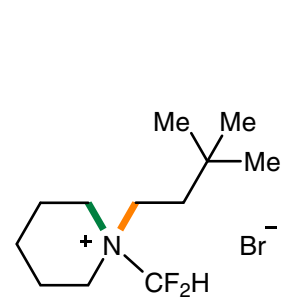

int3 $(0.0)$

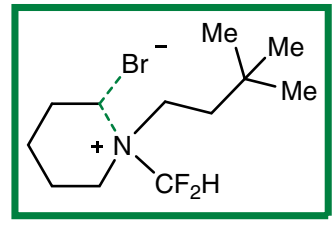

exclusive
Ring-opening TS

$(25.9 \mathrm{kcal} / \mathrm{mol})$

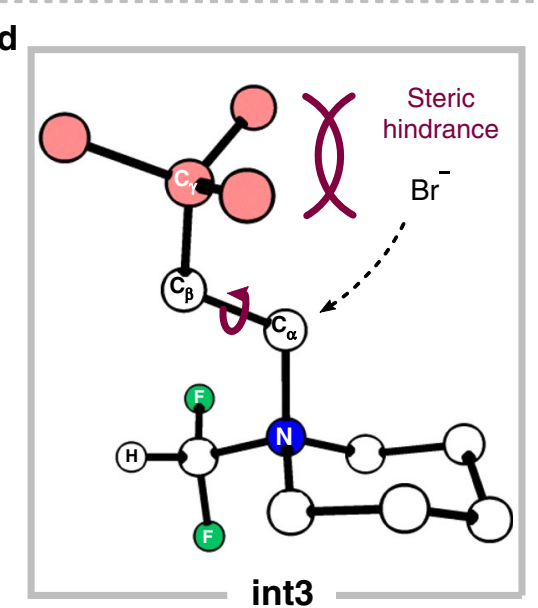

d
$\mathrm{N}$-dealkylation TS

$(30.4 \mathrm{kcal} / \mathrm{mol})$

$\Delta \Delta G^{\ddagger}=$

$4.6 \mathrm{kcal} / \mathrm{mol}$

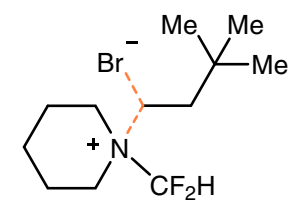

NOT allowed

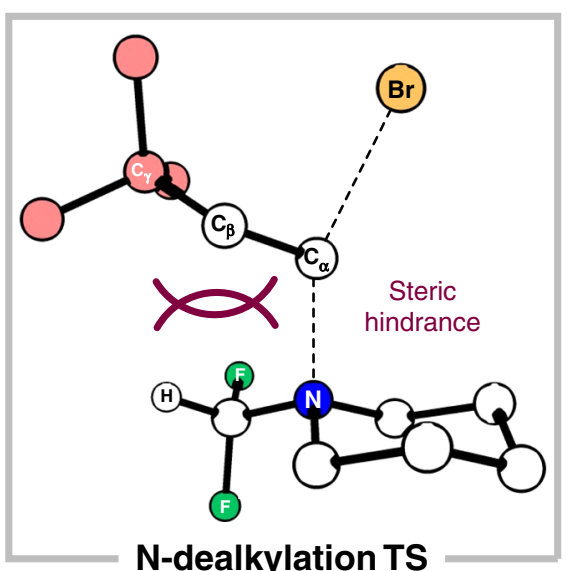

Fig. 5 Application to 6-membered cyclic amines and DFT calculations for the elucidation of the observed selectivity. a Influence of R group on the siteselectivity in 6-membered rings. b Competitive reaction energy profiles of $\mathrm{N}$-dealkylation and ring-opening pathways of $\mathrm{N}$-ethyl salt int2. c Competitive reaction energy profiles of $\mathrm{N}$-neohexyl salt int3. $\mathbf{d}$ Structural analysis for $\mathrm{N}$-dealkylation of int3. 
a

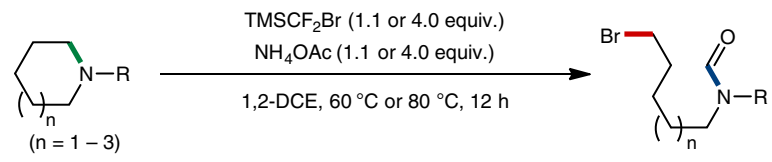

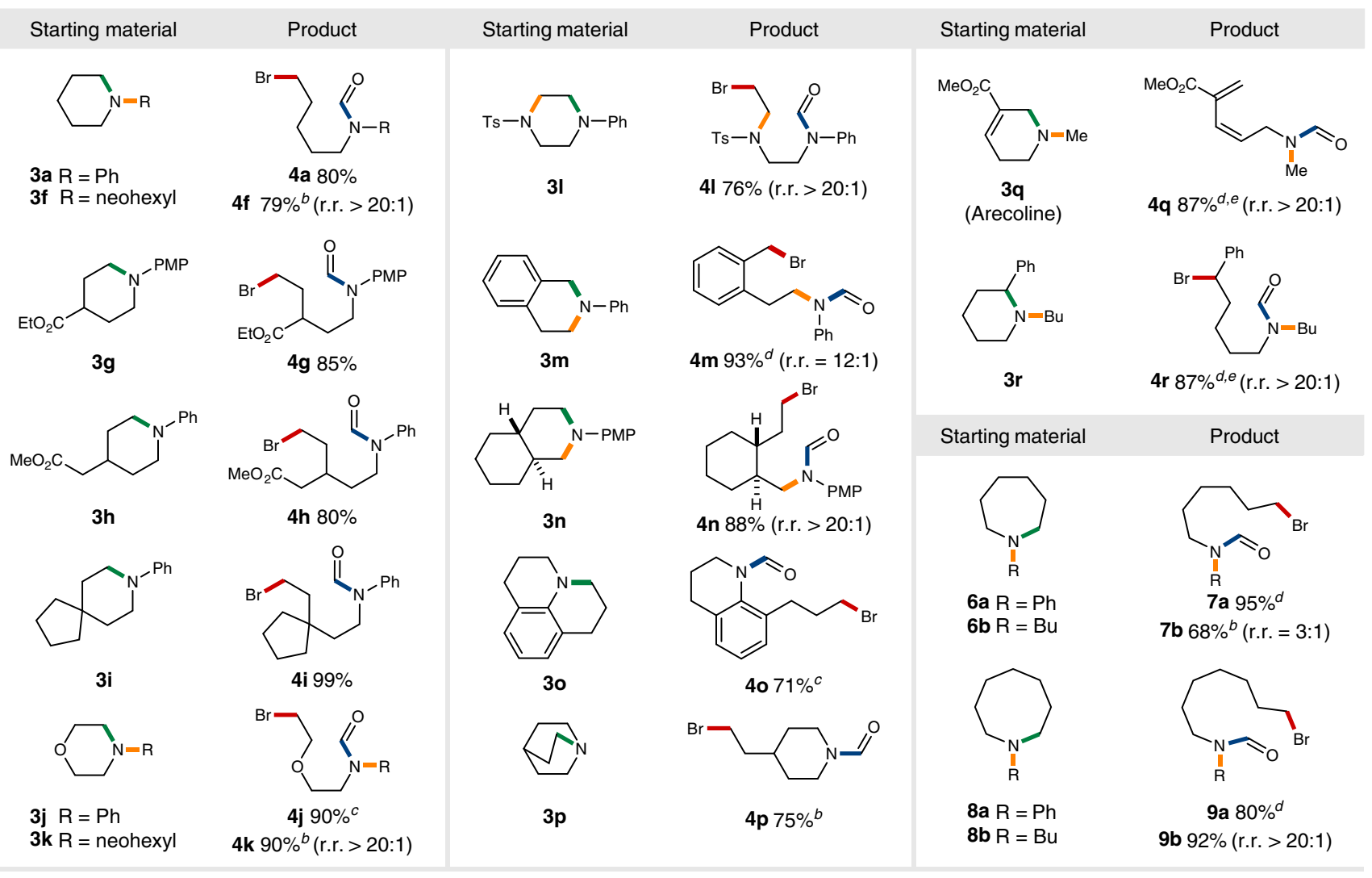<smiles>CCCc1nn(C)c2c(=O)[nH]c(-c3cc(S(=O)(=O)N4CCN(c5ccccc5)CC4)ccc3OCC)nc12</smiles>

$10 \mathrm{~N}$-Ph Sildenafil analogue I $\mathrm{TMSCF}_{2} \mathrm{Br}$ (4.0 equiv.) $\mathrm{NH}_{4} \mathrm{OAC}$ (4.0 equiv.) TCE, $60^{\circ} \mathrm{C}, 12 \mathrm{~h}$

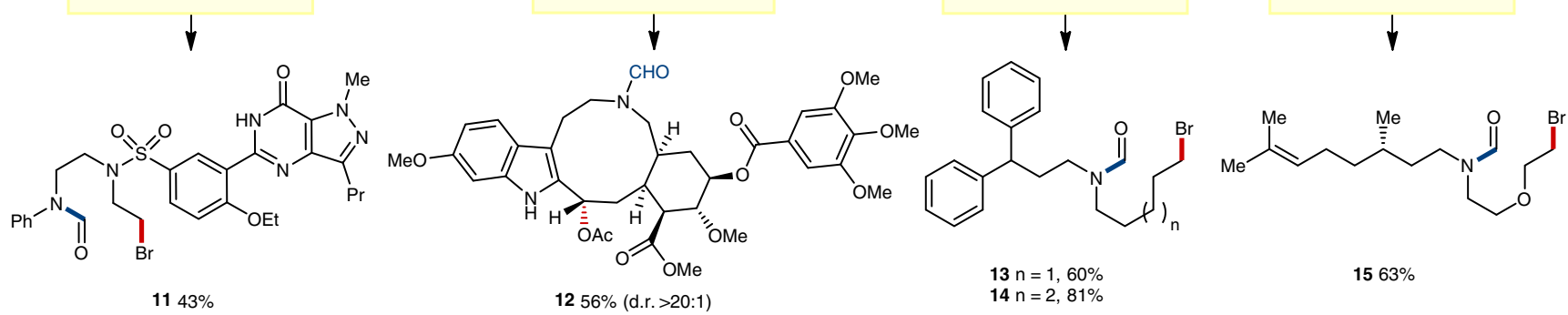

$1143 \%$

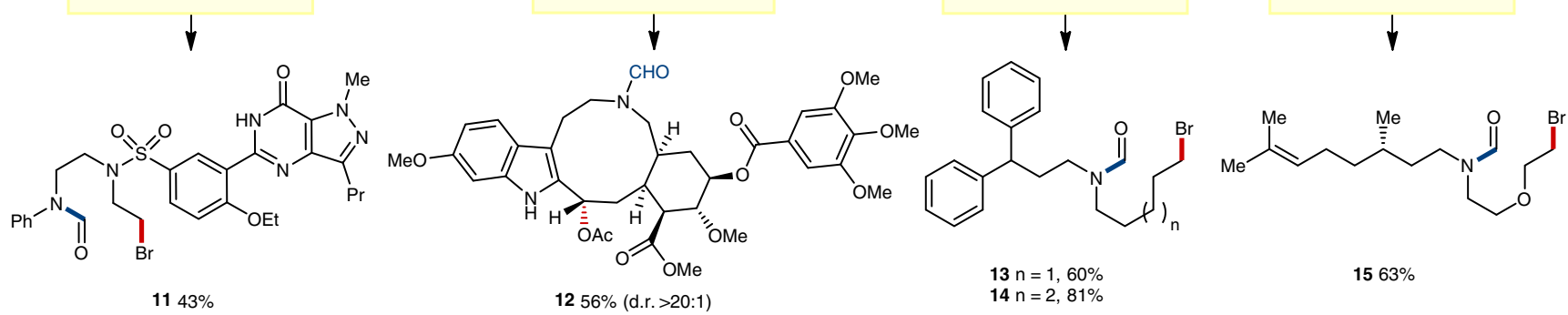<smiles>CCc1c[nH]c2cc(OC)ccc12</smiles>

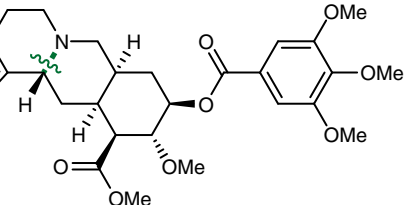
$\mathrm{OMe}^{\mathrm{OMe}}$ Reserpine I $\mathrm{TMSCF}_{2} \mathrm{Cl}$ (2.0 equiv.) $\mathrm{NH}_{4} \mathrm{OAC}$ (4.0 equiv.) 1,2-DCE, $25^{\circ} \mathrm{C}, 1 \mathrm{~h}$<smiles>C[N+]1(CCC(c2ccccc2)c2ccccc2)CCC2CCCCC21</smiles>

$\mathrm{n}=1$, Fenpiprane $\mathrm{n}=2$, Prozapine I

$\mathrm{TMSCF}_{2} \mathrm{Br}$ (4.0 equiv.) $\mathrm{NH}_{4} \mathrm{OAc}$ (4.0 equiv.) 1,2-DCE, $80^{\circ} \mathrm{C}, 12 \mathrm{~h}$<smiles>CC(C)=CCC[C@H](C)CC[NH+]1CCOCC1</smiles>

(S)-(+)-Citronellyl morpholine I

$\mathrm{TMSCF}_{2} \mathrm{Br}$ (4.0 equiv.) $\mathrm{NH}_{4} \mathrm{OAc}$ (4.0 equiv.) 1,2-DCE, $80^{\circ} \mathrm{C}, 12 \mathrm{~h}$

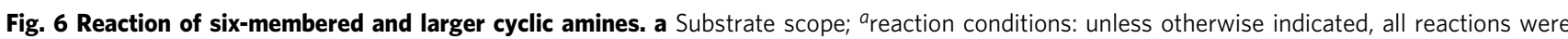
performed on a $0.2 \mathrm{mmol}$ scale using cyclic amine (1.0 equiv.), $\mathrm{TMSCF}_{2} \mathrm{Br}$ (4.0 equiv.), $\mathrm{NH}_{4} \mathrm{OAc}\left(4.0\right.$ equiv.) and 1,2-DCE (0.5 mL) at $60^{\circ} \mathrm{C}$. regioselectivity ratios (r.r.) were determined by ${ }^{1} \mathrm{H}$ NMR spectroscopy of crude mixtures. ${ }^{b} 80^{\circ} \mathrm{C} .{ }^{c} 40^{\circ} \mathrm{C}$. ${ }^{d} 25^{\circ} \mathrm{C}$. ${ }^{e} 1.1$ equiv. of $\mathrm{TMSCF}_{2} \mathrm{Br}$ and 1.1 equiv. of $\mathrm{NH}_{4} \mathrm{OAc}$. PMP para-methoxyphenyl. b Late-stage ring-opening functionalization of biologically relevant compounds.

regard, the ability to forge various ring structures through a common ring-opening approach would add a new dimension in generating structural-diversity. Taking advantages of the simultaneously introduced alkyl halide and $\mathrm{N}$-formyl groups in our products, we anticipated that a series of branching reactions would grant distinct skeletons.

First, one-pot sequence of lithium-iodine exchange, cyclization and reduction of the iodoformylation product $\mathbf{2 d - I}$ gave a 
a

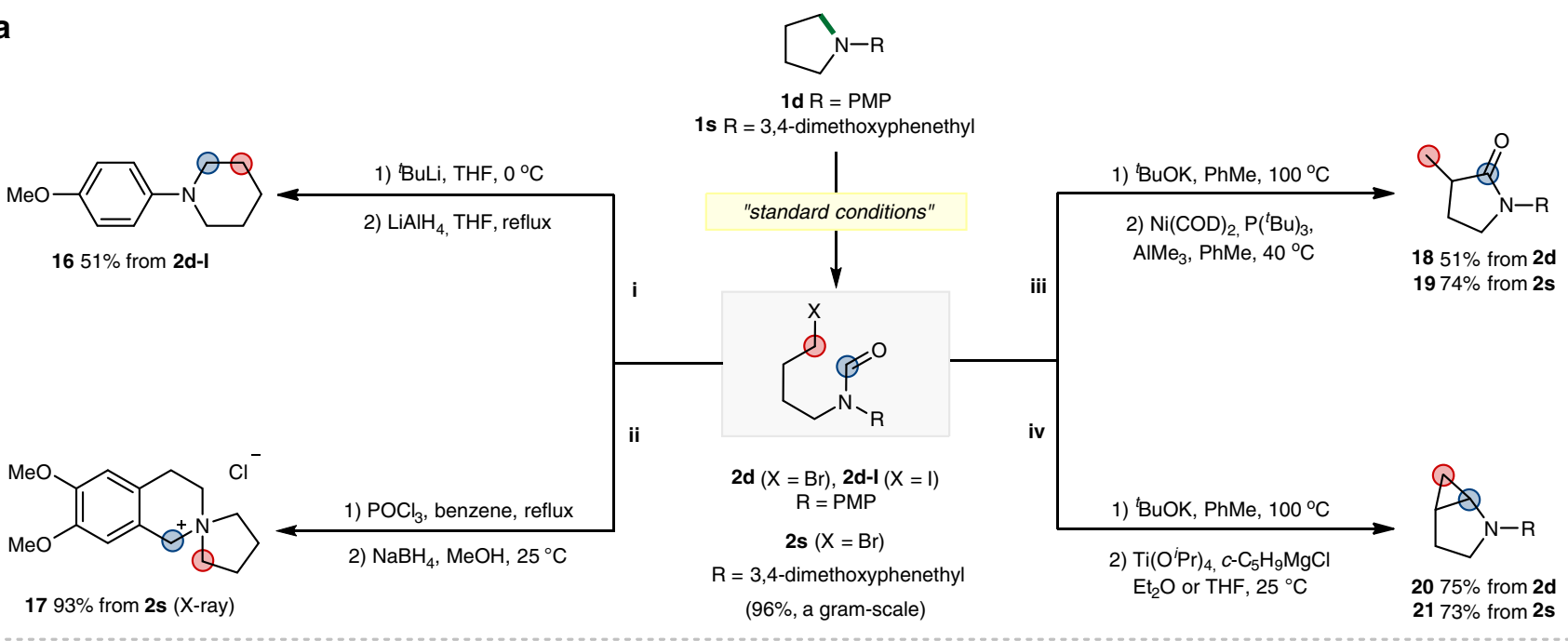

b

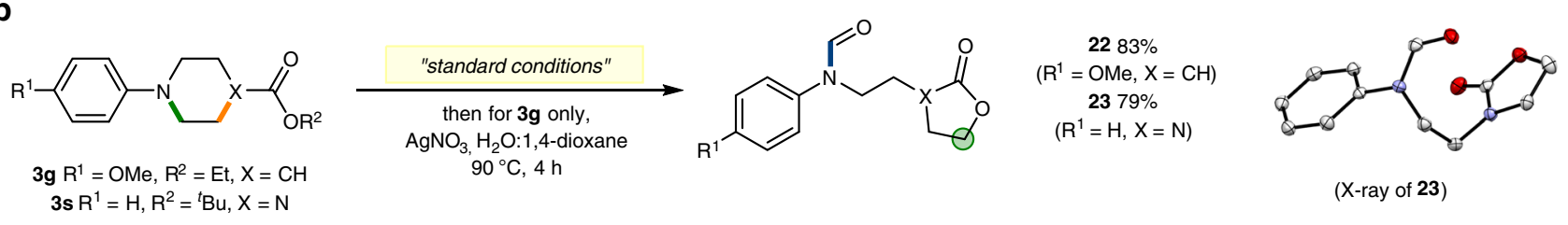

C

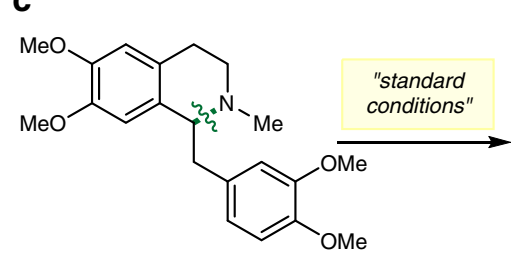

DL-Laudanosine<smiles>COc1ccc(/C=C/c2cc(OC)c(OC)cc2CCN(C=O)[N+](=O)[O-])cc1OC</smiles>

$2492 \%$

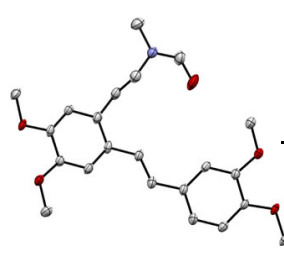

(X-ray of 24)

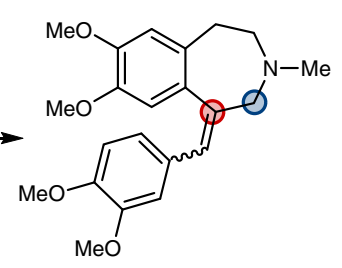

$2570 \%(E: Z=2: 1)$

Fig. 7 Skeletal diversification. a Skeletal remodelling of pyrrolidine rings. i, ring-expansion. ii, synthesis of spiro-bicyclic compound through the Bischler-Napieralski reaction. iii, synthesis of $\gamma$-lactam products by hydrocarbamoylation of E2 products. iv, synthesis of ring-fused products by the Kulinkovich-de Meijere reaction. b Skeletal remodelling of 6-membered rings by using a pre-installed nucleophile. c Ring-diversification of natural product: Ring-expansion of DL-Laudanosine.

ring-expansion product $\mathbf{1 6}$ in $51 \%$ yield (Fig. 7a, i). This ringopening-reclosing strategy is attractive in that it utilizes an external $\mathrm{C} 1$ source to achieve a homologation process that does not rely on the pre-encoded systems ${ }^{63}$. In the second ringmodification, 1-(3,4-dimethoxyphenethyl)pyrrolidine $1 \mathrm{~s}$ was ring-opened (2s, 96\%, a gram-scale) and subsequently treated under the Bischler-Napieralski reaction conditions to afford a C1-tethered product $\mathbf{1 7}$ in $93 \%$ yield (Fig. 7a, ii).

The conversion of the bromoalkyl group to olefin functionality via E2 elimination, and succeeding intramolecular reaction with the $\mathrm{N}$-formyl moiety offers another opportunity to obtain postmodification derivatives of high synthetic values. For example, Ni-catalysed hydrocarbamolylation ${ }^{64}$ of the newly generated double bond provided $\gamma$-lactam products 18 and 19 in 51 and $74 \%$ yields, respectively (Fig. $7 \mathrm{a}$, iii). In addition, Ti-mediated intramolecular cyclopropanation using the Kulinkovich-de Meijere conditions ${ }^{65}$ gave 2-azabicyclo[3.1.0] hexane products 20 and 21 in 75 and $73 \%$ yields from $2 d$ and 2 s, respectively (Fig. $7 a$, iv).

We next briefly examined skeletal remodelling based on the use of pre-installed functional groups (Fig. 7b). 4-Carboxylate substituted piperidine $\mathbf{3 g}$ was smoothly converted to a lactone product $22(83 \%)$ by a one-pot ring-opening, hydrolysis and cyclization procedure. Similarly, the reaction of $N$-Boc protected piperazine $3 \mathrm{~s}$ afforded a cyclic carbamate product $23(79 \%)$ under the standard ring-opening conditions, in this case, without the need of additional reagents. Moreover, the structure of obtained product 23 was characterized by its X-ray structure to confirm the site selectivity.

Furthermore, we demonstrated that our strategy could be utilized in ring-diversification of natural products. (Fig. 7c). For example, DL-Laudanosine was readily ring-opened by an elimination process to give the olefin intermediate 24 (92\%), and the subsequent Bischler-Napieralski reaction and hydride insertion led to a 7-membered analogue $\mathbf{2 5}$ in $70 \%$ yield. In addition to the excellent efficiency and selectivity achieved with biologically relevant compounds, this unique ability to create a diversified scaffold represents one of the distinguishing features of our current methodology.

In summary, we have developed an efficient strategy for deconstructive modification of unstrained azacyclic systems. The key finding was the use of difluorocarbene as a mild and practical source to form the key ammonium salt intermediates, which not only surpass the existing methodologies in terms of practicability, efficiency, functional group tolerance, chemo- and regioselecitvities, but also provides a unique tool to diversify a wider range of cyclic amines to acyclic analogues and homologated cyclic cores upon post-transformations. The utility of the developed protocol was further demonstrated by the direct structural alteration of azacyclic cores present within complex molecules. This late-stage 
diversification of natural products and pharmaceutical analogues enabled ready access to underexplored chemical space around bioactive molecules.

\section{Methods}

General procedure for the ring-opening bromoformylation. To a $2 \mathrm{~mL}$ reaction vial containing a mixture of $N$-phenylpyrrolidine $(29 \mu \mathrm{L}, 0.2 \mathrm{mmol})$ and $\mathrm{NH}_{4} \mathrm{OAc}$ $(61.7 \mathrm{mg}, 0.8 \mathrm{mmol})$ in 1,2 -dichloroethane $(1,2-\mathrm{DCE})(0.5 \mathrm{~mL})$ was added (bromodifluoromethyl)trimethylsilane $(124 \mu \mathrm{L}, 0.8 \mathrm{mmol})$. The vial was sealed and stirred at room temperature for $12 \mathrm{~h}$, then filtered through a pad of celite and washed with $\mathrm{CH}_{2} \mathrm{Cl}_{2}(5 \times 3 \mathrm{~mL})$. The filtrate was concentrated in vacuo, and the desired product was obtained as a colourless oil $(43 \mathrm{mg}, 85 \%)$ after purification by flash chromatography (eluent: EtOAc/n-Hexane, 1:5).

\section{Data availability}

All data generated and analysed during this study are included in this article and its Supplementary Information, and also available from the authors upon reasonable request. The X-ray crystallographic coordinates for structures reported in this article have been deposited at the Cambridge Crystallographic Data Centre (CCDC), under deposition numbers CCDC 1977901 (int1), 1977902 (23), 1977903 (17), 1977905 (24), 2000476 (int2) and 2000477 (int3). These data can be obtained free of charge via https:// www.ccdc.cam.ac.uk/structures/.

Received: 8 August 2020; Accepted: 27 August 2020;

Published online: 21 September 2020

\section{References}

1. Roughley, S. D. \& Jordan, A. M. The medicinal chemist's toolbox: an analysis of reactions used in the pursuit of drug candidates. J. Med. Chem. 54, 3451-3479 (2011).

2. Taylor, R. D., MacCoss, M. \& Lawson, A. D. G. Rings in drugs. J. Med. Chem. 57, 5845-5859 (2014).

3. Vitaku, E., Smith, D. T. \& Njardarson, J. T. Analysis of the structural diversity, substitution patterns, and frequency of nitrogen heterocycles among U.S. FDA approved pharmaceuticals. J. Med. Chem. 57, 10257-10274 (2014).

4. Blakemore, D. C. et al. Organic synthesis provides opportunities to transform drug discovery. Nat. Chem. 10, 383-394 (2018).

5. Beak, P., Kerrick, S. T., Wu, S. \& Chu, J. Complex induced proximity effects: enantioselective syntheses based on asymmetric deprotonations of $\mathrm{N}$-Bocpyrrolidines. J. Am. Chem. Soc. 116, 3231-3239 (1994).

6. Cordier, C. J., Lundgren, R. J. \& Fu, G. C. Enantioconvergent cross-couplings of racemic alkylmetal reagents with unactivated secondary alkyl electrophiles: catalytic asymmetric Negishi $\alpha$-alkylations of $N$-Boc-pyrrolidine. J. Am. Chem. Soc. 135, 10946-10949 (2013).

7. Shaw, M. H., Shurtleff, V. W., Terrett, J. A., Cuthbertson, J. D. \& MacMillan, D. W. C. Native functionality in triple catalytic cross-coupling: $\mathrm{sp}^{3} \mathrm{C}-\mathrm{H}$ bonds as latent nucleophiles. Science 352, 1304-1308 (2016).

8. Topczewski, J. J., Cabrera, P. J., Saper, N. I. \& Sanford, M. S. Palladium-catalysed transannular C-H functionalization of alicyclic amines. Nature 531, 220-224 (2016).

9. Chen, W., Paul, A., Abboud, K. A. \& Seidel, D. Rapid functionalization of multiple C-H bonds in unprotected alicyclic amines. Nat. Chem. 12, 545-550 (2020).

10. Walker, M. M. et al. Highly diastereoselective functionalization of piperidines by photoredox-catalyzed $\alpha$-amino C-H arylation and epimerization. J. Am. Chem. Soc. 142, 8194-8202 (2020).

11. Karageorgis, G., Foley, D. J., Laraia, L. \& Waldmann, H. Principle and design of pseudo-natural products. Nat. Chem. 12, 227-235 (2020).

12. Ouyang, K., Hao, W., Zhang, W.-X. \& Xi, Z. Transition-metal-catalyzed cleavage of C-N single bonds. Chem. Rev. 115, 12045-12090 (2015).

13. Wang, Q., Su, Y., Li, L. \& Huang, H. Transition-metal catalysed C-N bond activation. Chem. Soc. Rev. 45, 1257-1272 (2016).

14. Souillart, L. \& Cramer, N. Catalytic C-C bond activations via oxidative addition to transition metals. Chem. Rev. 115, 9410-9464 (2015).

15. Murakami, M. \& Ishida, N. Potential of metal-catalyzed C-C single bond cleavage for organic synthesis. J. Am. Chem. Soc. 138, 13759-13769 (2016).

16. $\mathrm{Xu}, \mathrm{Y}$. et al. Deacylative transformations of ketones via aromatizationpromoted C-C bond activation. Nature 567, 373-378 (2019).

17. Roque, J. B., Kuroda, Y., Göttemann, L. T. \& Sarpong, R. Deconstructive fluorination of cyclic amines by carbon-carbon cleavage. Science 361, 171-174 (2018).

18. Leonard, D. K., Li, W., Junge, K. \& Beller, M. Improved bimetallic cobalt-manganese catalysts for selective oxidative cleavage of morpholine derivatives. ACS Catal. 9, 11125-11129 (2019).
19. Romero-Ibañez, J. et al. Transition-metal-free deconstructive lactamization of piperidines. Angew. Chem. Int. Ed. 58, 8867-8871 (2019).

20. $\mathrm{Li}$, W. et al. Practical catalytic cleavage of $\mathrm{C}\left(\mathrm{sp}^{3}\right)-\mathrm{C}\left(\mathrm{sp}^{3}\right)$ bonds in amines. Angew. Chem. Int. Ed. 58, 10693-10697 (2019).

21. Louwrier, S., Tuynman, A. \& Hiemstra, H. Synthesis of bicyclic guanidines from pyrrolidin-2-one. Tetrahedron 52, 2629-2646 (1996).

22. Van Betsbrugge, J., Van Den Nest, W., Verheyden, P. \& Tourwé, D. New amino acids derived from L-pyroglutamic acid: synthesis of trans-4-benzylcis-5-phenyl-L-proline, L- $\alpha$-(2-benzyl-3-phenylpropyl)-glycine and L- $\alpha$-(3phenylpropyl)-glycine. Tetrahedron 54, 1753-1762 (1998).

23. Honda, T. \& Ishikawa, F. Reductive deamination of $\alpha$-amino carbonyl compounds by means of samarium iodide. Chem. Commun. 1065-1066 (1999).

24. Azzena, U., Demartis, S., Pilo, L. \& Piras, E. Reductive cleavage of benzannelated cyclic ethers and amines: synthetic applications. Tetrahedron 56, 8375-8382 (2000).

25. Schreiber, S. L. Target-oriented and diversity-oriented organic synthesis in drug discovery. Science 287, 1964-1969 (2000).

26. Wender, P. A., Verma, V. A., Paxton, T. J. \& Pillow, T. H. Function-oriented synthesis, step economy, and drug design. Acc. Chem. Res. 41, 40-49 (2008)

27. Galloway, W. R. J. D., Isidro-Llobet, A. \& Spring, D. R. Diversity-oriented synthesis as a tool for the discovery of novel biologically active small molecules. Nat. Commun. 1, 80 (2010).

28. Castaño, A. M., Cuerva, J. M. \& Echavarren, A. M. A concise synthesis of $( \pm)$-monomorine I by way of a palladium-catalyzed reductive coupling. Tetrahedron Lett. 35, 7435-7438 (1994).

29. Cocquet, G., Ferroud, C. \& Guy, A. A mild and efficient procedure for ringopening reactions of piperidine and pyrrolidine derivatives by single electron transfer photooxidation. Tetrahedron 56, 2975-2984 (2000).

30. Ito, R., Umezawa, N. \& Higuchi, T. Unique oxidation reaction of amides with pyridine- $N$-oxide catalyzed by ruthenium porphyrin: direct oxidative conversion of $\mathrm{N}$-acyl-L-proline to $\mathrm{N}$-Acyl-L-glutamate. J. Am. Chem. Soc. 127, 834-835 (2005).

31. Liu, P., Liu, Y., Wong, E. L.-M., Xiang, S. \& Che, C.-M. Iron oligopyridine complexes as efficient catalysts for practical oxidation of arenes, alkanes, tertiary amines and $\mathrm{N}$-acyl cyclic amines with Oxone. Chem. Sci. 2, 2187-2195 (2011)

32. Osberger, T. J., Rogness, D. C., Kohrt, J. T., Stepan, A. F. \& White, M. C. Oxidative diversification of amino acids and peptides by small-molecule iron catalysis. Nature 537, 214-219 (2016).

33. Roque, J. B., Kuroda, Y., Göttemann, L. T. \& Sarpong, R. Deconstructive diversification of cyclic amines. Nature 564, 244-248 (2018).

34. Cope, A. C. \& Trumbull, E. R. Olefins from amines: the Hofmann elimination reaction and amine oxide pyrolysis. Org. React. 11, 317-493 (1960).

35. Zhu, Q., Yuan, Q., Chen, M., Guo, M. \& Huang, H. Multicomponent reactions with cyclic tertiary amines enabled by facile $\mathrm{C}-\mathrm{N}$ bond cleavage. Angew Chem. Int. Ed. 56, 5101-5105 (2017).

36. Ross, S. P. \& Hoye, T. R. Reactions of hexadehydro-Diels-Alder benzynes with structurally complex multifunctional natural products. Nat. Chem. 9, 523-530 (2017).

37. Bugaenko, D. I., Yurovskaya, M. A. \& Karchava, A. V. N-Arylation of DABCO with diaryliodonium salts: general synthesis of $N$-aryl-DABCO salts as precursors for 1,4-disubstituted piperazines. Org. Lett. 20, 6389-6393 (2018).

38. Sim, T. B. et al. A novel synthesis of 5-functionalized oxazolidin-2-ones from enantiomerically pure 2 -substituted $N-[(R)-(+)-\alpha$-methylbenzyl $]$ aziridines. $J$. Org. Chem. 68, 104-108 (2003).

39. Hu, X. E. Nucleophilic ring opening of aziridines. Tetrahedron 60, 2701-2743 (2004).

40. Couty, F. et al. Nucleophilic ring-opening of azetidinium ions: insights into regioselectivity. Eur. J. Org. Chem. 2006, 3479-3490 (2006).

41. Vargas-Sanchez, M., Lakhdar, S., Couty, F. \& Evano, G. Reaction of azetidines with chloroformates. Org. Lett. 8, 5501-5504 (2006).

42. Wright, K., Drouillat, B., Menguy, L., Marrot, J. \& Couty, F. The von Braun reaction applied to azetidines. Eur. J. Org. Chem. 2017, 7195-7201 (2017).

43. von Braun, J. Die einwirkung von bromcyan auf tertiäre amine. Ber. Dtsch. Chem. Ges. 33, 1438-1452 (1900).

44. Hageman, H. A. The von Braun cyanogen bromide reaction. Org. React. 7, 198-262 (1953).

45. Elderfield, R. C. \& Hageman, H. A. The von Braun cyanogen bromide reaction I. application to pyrrolidines and ethylenimines (1). J. Org. Chem. 14, 605-637 (1949).

46. Magnier, E., Langlois, Y. \& Mérienne, C. Stereoselective synthesis of the tricyclic core of manzamine a: the bradsher cycloaddition route. Tetrahedron Lett. 36, 9475-9478 (1995).

47. Paciaroni, N. G. et al. A Tryptoline ring-distortion strategy leads to complex and diverse biologically active molecules from the indole alkaloid Yohimbine. Chem. Eur. J. 23, 4327-4335 (2017).

48. Seong, S., Lim, H. \& Han, S. Biosynthetically inspired transformation of iboga to monomeric post-iboga alkaloids. Chem 5, 353-363 (2019). 
49. Glotz, G., Lebl, R., Dallinger, D. \& Kappe, C. O. Integration of bromine and cyanogen bromide generators for the continuous-flow synthesis of cyclic guanidines. Angew. Chem. Int. Ed. 56, 13786-13789 (2017).

50. Rice, K. C. Improved procedure for the $N$-demethylation of $6,7-$ benzomorphans, morphine, and codeine. J. Org. Chem. 40, 1850-1851 (1975).

51. Olofson, R. A., Schnur, R. C., Bunes, L. \& Pepe, J. P. Selective N-dealkylation of tertiary amines with vinyl chloroformate: an improved synthesis of naloxone. Tetrahedron Lett. 18, 1567-1570 (1977).

52. Kapnang, H. \& Charles, G. Reaction des chloroformiates sur les amines tertiaires: competition entre $\mathrm{N}$-demethylation, desamination, $\mathrm{N}$-debenzylation et $N$-desallylation. Tetrahedron Lett. 24, 3233-3236 (1983).

53. Olofson, R. A., Martz, J. T., Senet, J. P., Piteau, M. \& Malfroot, T. A new reagent for the selective, high-yield $N$-dealkylation of tertiary amines: improved syntheses of naltrexone and nalbuphine. J. Org. Chem. 49, 2081-2082 (1984).

54. Bhat, R. G., Ghosh, Y. \& Chandrasekaran, S. A mild and selective method for $\mathrm{N}$-dealkylation of tertiary amines. Tetrahedron Lett. 45, 7983-7985 (2004).

55. Yu, C. et al. Selective ring-opening of $N$-alkyl pyrrolidines with chloroformates to 4-chlorobutyl carbamates. J. Org. Chem. 82, 6615-6620 (2017).

56. Ni, C. \& Hu, J. Recent advances in the synthetic application of difluorocarbene. Synthesis 46, 842-863 (2014).

57. Dilman, A. D. \& Levin, V. V. Difluorocarbene as a building block for consecutive bond-forming reactions. Acc. Chem. Res. 51, 1272-1280 (2018).

58. Li, L., Wang, F., Ni, C. \& Hu, J. Synthesis of gem-difluorocyclopropa(e)nes and $\mathrm{O}-, \mathrm{S}-, \mathrm{N}-$, and P-difluoromethylated compounds with $\mathrm{TMSCF}_{2} \mathrm{Br}$. Angew. Chem. Int. Ed. 52, 12390-12394 (2013).

59. Ma, X., Deng, S. \& Song, Q. Halodifluoroacetates as formylation reagents for various amines via unprecedented quadruple cleavage. Org. Chem. Front. 5, 3505-3509 (2018).

60. Wuts, P. G. \& Greene, T. W. Greene's Protective Groups in Organic Synthesis. 4th edn (John Wiley \& Sons, Inc., New Jersey, 2007).

61. Yonovich, M. \& Sasson, Y. Conversion of tertiary amines to formamides in presence of dichlorocarbene and phenoxide ion. Tetrahedron Lett. 21 1875-1876 (1980).

62. DeTar, D. F., McMullen, D. F. \& Luthra, N. P. Steric effects in $\mathrm{S}_{\mathrm{N}} 2$ reactions. J. Am. Chem. Soc. 100, 2484-2493 (1978).

63. Cossy, J., Dumas, C. \& Pardo, D. G. Ring expansion-formation of optically active 3-hydroxypiperidines from pyrrolidinemethanol derivatives. Eur. J. Org. Chem. 1999, 1693-1699 (1999).

64. Nakao, Y., Idei, H., Kanyiva, K. S. \& Hiyama, T. Hydrocarbamoylation of unsaturated bonds by nickel/Lewis-acid catalysis. J. Am. Chem. Soc. 131, 5070-5071 (2009).

65. de Meijere, A., Kozhushkov, S. I. \& Savchenko, A. I. Titanium-mediated syntheses of cyclopropylamines. J. Organomet. Chem. 689, 2033-2055 (2004).

\section{Acknowledgements}

Financial support for this research was provided by the Institute for Basic Science (IBSR010-D1 and IBS-R010-Y2) in South Korea.

\section{Author contributions}

S.C. and S.S. conceived, designed and organized the project. Y.K. and S.S. performed the experiments. Y.K. and J.H. conducted the DFT calculations. D.K. analysed the X-ray crystal structures. Y.K., J.H., S.C. and S.S. co-wrote the paper. All authors analysed the data, discussed the results and commented on the paper.

\section{Competing interests}

The authors declare no competing interests.

\section{Additional information}

Supplementary information is available for this paper at https://doi.org/10.1038/s41467020-18557-8.

Correspondence and requests for materials should be addressed to S.C. or S.S.

Peer review information Nature Communications thanks the anonymous reviewer(s) for their contribution to the peer review of this work.

Reprints and permission information is available at http://www.nature.com/reprints

Publisher's note Springer Nature remains neutral with regard to jurisdictional claims in published maps and institutional affiliations.

(c) (i) Open Access This article is licensed under a Creative Commons Attribution 4.0 International License, which permits use, sharing, adaptation, distribution and reproduction in any medium or format, as long as you give appropriate credit to the original author(s) and the source, provide a link to the Creative Commons license, and indicate if changes were made. The images or other third party material in this article are included in the article's Creative Commons license, unless indicated otherwise in a credit line to the material. If material is not included in the article's Creative Commons license and your intended use is not permitted by statutory regulation or exceeds the permitted use, you will need to obtain permission directly from the copyright holder. To view a copy of this license, visit http://creativecommons.org/ licenses/by/4.0/.

(C) The Author(s) 2020 